\title{
Thermodynamics in landscape ecology: the importance of integrating measurement and modeling of landscape entropy
}

\author{
Samuel A. Cushman
}

Received: 2 August 2014 / Accepted: 19 October 2014/Published online: 6 November 2014

(C) Springer Science+Business Media Dordrecht (outside the USA) 2014

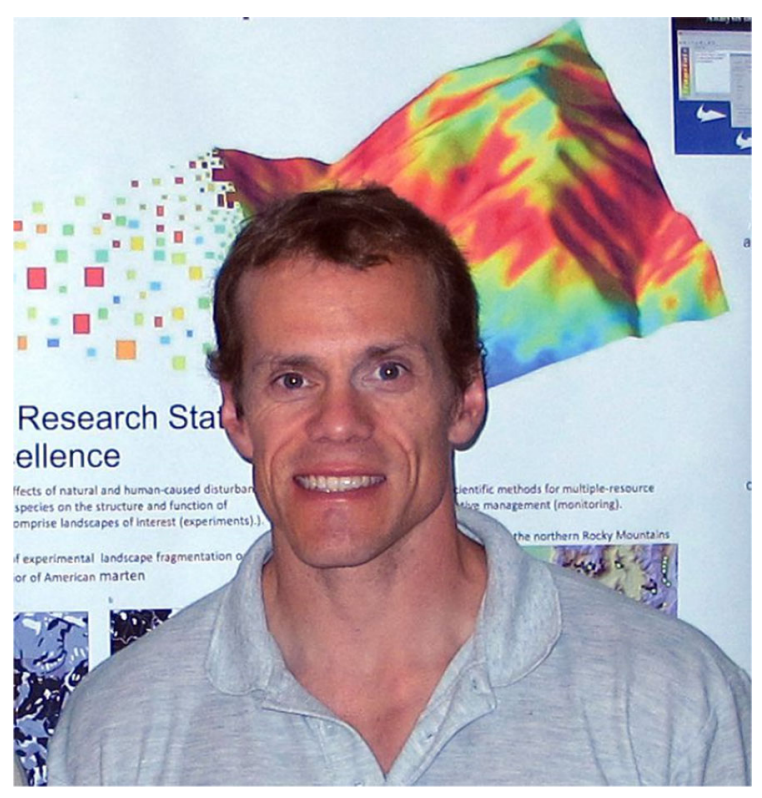

Entropy and the second law of thermodynamics are the central organizing principles of nature. Or perhaps more accurately, the second law is the central disorganizing principle. Hot things cool down. Cold things warm up. You cannot get something for nothing. You always pay more than you get. Things fall apart. You

S. A. Cushman ( $ه)$

USDA Forest Service, Rocky Mountain Research Station, 2500 S Pine Knoll Dr., Flagstaff, AZ 86001, USA

e-mail: scushman@fs.fed.us cannot repeat the past. We grow old and die. It is all downhill from here to the heat death of the universe. While at first these seem like grim and pessimistic ideas, a deeper understanding reveals that the disequilibrial processes of increasing entropy are what enables all organizing actions, such as the formation of a molecule, building of a cell, birth of a child, organization of an ecosystem. Yet strangely, the ideas and implications of the second law are poorly developed in the landscape ecology literature. This is particularly strange given the focus of landscape ecology on understanding pattern-process relationships across scales in space and time. Every interaction between entities leads to irreversible change which increases the entropy and decreases the free energy of the closed system in which they reside. This is the essence of the entropy principle. Descriptions of landscape patterns, processes of landscape change, propagation of pattern-process relationships across space and through time are all governed, constrained, and in large part directed by thermodynamics. This direct linkage to thermodynamics and entropy was noted in several of the pioneering works in the field of landscape ecology (e.g. Forman and Godron 1986; O'Neill et al. 1986; Naveh 1987; O'neill et al. 1989). Yet in the subsequent decades our field has largely failed to embrace and utilize these relationships and constraints, with a few exceptions (see Wu and Loucks 1995; Zhang and Wu 2002; Zurlini et al. 2013).

Landscape ecology sometimes has been criticized as a descriptive science focused on patterns without 
linkage to central organizing theories or principles. In recent decades this has been reversed in part with strong landscape ecology research focused directly on pattern-process relationships, drivers and responses across scales in space and time. Landscape ecology is the science of understanding the interactions of patterns and processes across scales. The second law of thermodynamics and the entropy principle provide a theoretical context which could help clarify and unify a large portion of landscape ecology research, and connect it to fundamental principles.

In a review paper published in this issue Vranken et al. (2014) present an overview of the use of entropy in landscape ecology. They identified three main uses of the entropy concept in past landscape ecology research, including: spatial heterogeneity, unpredictability of pattern dynamics, and pattern dependence on scale. They conclude from their review that thermodynamic interpretations of spatial heterogeneity in the literature are not relevant, that thermodynamic interpretations related to scale dependence are highly questionable, and that of all applications of entropy in landscape ecology only unpredictability could be thermodynamically relevant if appropriate measurements were performed to test it. They note that while entropy is frequently mentioned in landscape ecology literature, it is rarely formally addressed and usually only applied in imprecise and descriptive terms. They note frequent contradictions in the interpretation of entropy in landscape ecology literature.

The Vranken et al. (2014) review, in my opinion, shows how poorly the universally important topics of the second law of thermodynamics and the entropy principle have penetrated landscape ecology. There are many examples in the literature of informal linkage to the entropy concept, but virtually no formal, quantitative efforts to develop explicit theory derived from the second law. The review shows very few examples of the concepts even being mentioned (as a proportion of the literature) and virtually no formal, quantitative, theoretically justifiable application of thermodynamic ideas. They note that most authors addressing linkages between entropy and landscape patterns generally use the entropy principle metaphorically, referring to the linkage between entropy and landscape disorder, but not formally calculating it. Further they note contradictory interpretations of the conceptual linkage between entropy and landscape pattern. They note that no formal proposal for calculating the thermodynamic entropy of landscapes has been published and as a result conclude that any link between spatial heterogeneity and thermodynamic entropy should be treated with caution. This seems astounding for a field that has been so obsessed with measuring and interpreting landscape patterns has entirely neglected the fundamentally important and interesting task of measuring the entropy of a landscape mosaic, and connecting it more broadly to understanding of pattern-process relationships. Such a formal analysis and definition of landscape entropy may facilitate understanding the linkages between landscape pattern-process relationships and entropy and the second law of thermodynamics. Such a definition and linkage is necessary for using thermodynamic principles to understand and predict landscape dynamics and thermodynamic controls on the propagation of pattern-process relationships across scales, which are other areas Vranken et al. (2014) identify as topics mentioned in past landscape ecology literature, but that are incoherently and inconsistently presented due to lack of a rigorous theoretical framework in which to analyze and interpret data on these topics.

Below are some ideas that I hope will provoke discussion and rekindle research on the linkages between the second law of thermodynamics, the entropy principle, landscape patterns, and the propagation of pattern-process relationships across space and through time.

- There is a critical need to define the configurational entropy of landscape mosaics as a benchmark and measuring stick which subsequently can be used to quantify entropy changes in landscape dynamics and the interactions of patterns and processes across scales of space and time.

- The second law and entropy are of direct relevance to landscape dynamics as all changes in nature result in increases in system disorder and reduction in free energy of the closed system. Therefore landscape time series data record this process. In a closed system all time series will show increasing disorder and reduction in free energy over time, but ecological systems are open systems, and thus time series may show dynamic patterns without directional changes in disorder or free energy. Most importantly, ecological systems are driven by continual inflow of energy from the sun, and 
absolutely not thermodynamically closed systems. This inflow of energy enables biological processes to function, driving photosynthesis "uphill" against the current of entropy, with ecological food webs then providing a "cascade" back down the free energy ladder, reducing free energy and increasing thermodynamic disorder. Landscape ecologists should more formally associate landscape dynamics with changes in entropy and quantify the function of ecological dissipative structures.

- Observing a dynamic equilibrium in a landscape does not imply absence of increasing entropy. Just as an organism maintains homeostasis by functioning as a dissipative structure consuming and degrading high free energy organic molecules and releasing heat and highly oxidized metabolic products, a landscape maintains a dynamic equilibrium under a disturbance-succession regime through the collective emergent property of many organismal dissipative structures in interaction with abiotic drivers such as solar energy, temperature, and moisture.

- In forest systems the dynamics range from gapphase replacement of individual trees as windfall and senescence occurs to large scale patterns of patch dynamics in response to wild fire and other large contagious disturbances (e.g. Wu and Loucks 1995). In each of these there is a dynamic equilibrium of landscape patterns, with different kinds of heterogeneity at different scales. In neither is there any trend to decrease in macrostructural stage, but rather a characteristic range of variation in landscape structure over time (e.g., change in macrostate within a characteristic range), as a function of the nature of the disturbance-succession process in that system. Linking the scale dependence of landscape dynamics to thermodynamic constraints across different ecosystem types would be central to generalizing the application of entropy in landscape ecology.

- The linkage of the second law of thermodynamics and the entropy principle with the concepts of resistance, resilience and recovery seems important, as is linkage to ideas of dynamic equilibrium and dissipative structures.

- There are more ways to be broken than to be fixed, more ways to be dead than alive, more ways to be disordered than to be ordered, and thus thermodynamic changes always lead to less predictability in the future state than the current state. All increases in entropy result in increasing disorder and lower potential energy in the closed system. This by definition decreases predictability, as there are more ways to be disordered than ordered and more ways to have dissipated energy than "concentrated" energy. This is always the case, and increase in entropy always leads to decrease in predictability in the closed system. However, landscapes are open systems and understanding the flow of energy and resulting patterns of order and disorder may result in increase or decrease in system predictability over time depending on whether the energy flow results in net decrease in entropy of the landscape or a net increase.

- Fractal dimension seems directly related to entropy. Fractal dimension is a measure of a pattern-process scaling law and the relationships of such scaling laws to the thermodynamics of dissipative structures is a topic that should be explored. One may conjecture that the reason there are fractal scaling laws at all is because of the thermodynamic behavior of dissipative structures.

- The scale challenges in landscape ecology are not a source of "departure" from thermodynamics, but rather are products of the action of dissipative structures organized across a range of scales or hierarchical levels (e.g. O'Neill et al. 1986; Wu and Loucks 1995). Attention should be given to linkages between entropy, complexity theory and the organization of ecological systems as a multilevel or multi-scale system of dissipative structures.

- Thermodynamic irreversibility is a fundamental attribute of the universe and all things in it, including landscapes. If landscapes appear to not follow irreversibility laws then it is an indication of an insufficiency of how landscape ecological analysis reflects the reality of the universe. When ecological systems are properly viewed as multiscale and hierarchically organized dissipative structures then it is clear that thermodynamic irreversibility does apply.

- The application of thermodynamic entropy concepts in landscape ecology has not addressed the true thermodynamic nature of the actions of dissipative structures across scales, and this has 
been limited by failure to measure energy transformations, changes in free energy, changes in configurational entropy of landscape mosaics. As a result, there has been a nebulous and inconsistent application and interpretation of these ideas in the field.

Acknowledgements I would like to thank Susana Rostro for encouraging me to revisit these long simmering ideas and finally put them into text for publication.

\section{References}

Forman RTT, Godron M (1986) Heterogeneity and typology. In: Forman RTT, Godron M (eds) Landscape ecology. Wiley, New York, pp 463-493

Naveh Z (1987) Biocybernetic and thermodynamic perspectives of landscape functions and land use patterns. Landscape Ecol 1:75-83
O’Neill RV, DeAngelis DL, Waide JB, Allen TFH (1986) A hierarchical concept of ecosystems. Princeton University Press, Princeton

O'neill RV, Johnson AR, King AW (1989) A hierarchical framework for the analysis of scale. Landscape Ecol 3:13

Vranken I, Baudry J, Aubinet M, Visser M, Bogaert J (2014) A review on the use of entropy in landscape ecology: heterogeneity, unpredictability, scale dependence and their links with thermodynamics. Landscape Ecology (this issue)

Wu JG, Loucks OL (1995) Fromblanace of nature to hierarchical patch dynamics: a paradigm shift in ecology. Q Rev Biol 70:439-466

Zhang H, Wu JG (2002) A statistical thermodynamic model of the organizational order of vegetation. Ecol Model 153:69-80

Zurlini G, Petrosillo I, Jones B, Zaccarelli N (2013) Highlighting order and disorder in social-ecological landscapes to foster adaptive capacity and sustainability. Landscape Ecol 28:1161-1173 